\title{
Abundances of planetary nebulae NGC 3242 and NGC 6369^
}

\author{
S. R. Pottasch ${ }^{1}$ and J. Bernard-Salas ${ }^{2}$ \\ 1 Kapteyn Astronomical Institute, PO Box 800, 9700 Av. Groningen, The Netherlands \\ e-mail: pottasch@astro.rug.nl \\ 2 Center for Radiophysics and Space Research, Cornell University, Ithaca, NY 14853, USA
}

Received 31 July 2008 / Accepted 12 September 2008

\section{ABSTRACT}

\begin{abstract}
The spectra of the planetary nebulae NGC 3242 and NGC 6369 are reanalysed using spectral measurements made in the mid-infrared with the Spitzer Space Telescope and the Infrared Space Observatory (ISO). The aim is to determine the chemical composition of these objects. We also make use of International Ultraviolet Explorer (IUE) and ground based spectra. These elliptical PNe are interesting because they are well-studied, nearby, bright objects and therefore allow a reasonably complete comparison of this type of nebulae. Abundances determined from the mid-infrared lines, which are insensitive to electron temperature, are used as the basis for the determination of the composition, which are found to differ somewhat from earlier results. The abundances found, especially the low value of helium and oxygen, indicate that the central star was originally of rather low mass. The abundance of phosphorus has been determined for the first time in NGC 3242. The electron temperature in both of these nebulae is roughly constant unlike NGC 6302 and NGC 2392 where a strong temperature gradient is found. The temperature of the central star is discussed for both nebulae. Finally a comparison of the element abundances in these nebulae with the solar abundance is made. The low abundance of $\mathrm{Fe}$ and $\mathrm{P}$ is noted and it is suggested that these elements are an important constituent of the nebular dust.
\end{abstract}

Key words. ISM: abundances - ISM: planetary nebulae: individual: NGC 3242, NGC 6369 - infrared: ISM

\section{Introduction}

NGC 3242 (PK 261.0+32.0) is a bright planetary nebula with a low radial velocity and is located at a rather high galactic latitude. The nebula has a bright inner ellipsoidal shell of about $28^{\prime \prime} \times 20^{\prime \prime}$. This is surrounded by an almost spherical halo of $46^{\prime \prime} \times 40^{\prime \prime}$ with considerably lower emission. Both the inner and outer regions contain little structure. The nebula is located about 32 degrees above the galactic plane and has little or no extinction. Because of its brightness it is clear that it is a nearby nebula. An expansion distance is known for this nebula to be about 0.5 kpc (Terzian 1997; Mellema 2004).

The nebula has a rather bright central star $(V=12.43)$ which has been studied by several authors. Pauldrach et al. (2004) have compared the stellar spectrum with a model atmosphere and conclude that the star has an effective temperature $T_{\text {eff }}=75000 \mathrm{~K}$ which is in between the hydrogen Zanstra temperature of about $59000 \mathrm{~K}$, and somewhat less than the ionized helium Zanstra temperature which is close to $91000 \mathrm{~K}$ (Gathier \& Pottasch 1988). Tinkler \& Lamers (2002) prefer to assign a higher effective temperature of $T_{\mathrm{eff}}=94000 \mathrm{~K}$. to the star based on the high ionized helium Zanstra temperature. We shall discuss the stellar temperature later in the paper.

NGC 6369 (PK 002.4+05.8) is located in the direction of the galactic bulge but it is undoubtably a nearby $\mathrm{PN}$ for two reasons. First, it is a rather large nebula, with a diameter of about $32^{\prime \prime}$. Second, it is the third brightest PN in the sky, at least at radio frequencies. It cannot however be very close because the extinction

^ Based on observations with the Spitzer Space Telescope, which is operated by the Jet Propulsion Laboratory, California Institute of Technology. is quite high and its radial velocity is also high $\left(-101 \mathrm{~km} \mathrm{~s}^{-1}\right)$. The nebula can be described as a bright ring with an outer diameter of $32.4^{\prime \prime}$ and an inner diameter of about $13^{\prime \prime}$. There is faint emission both to the east and the west of the main structure. The central star is clearly visible. It is classified as spectral type WC4 and has a magnitude $V=15.91$ (Gathier \& Pottasch 1988). Its hydrogen Zanstra temperature is about $70000 \mathrm{~K}$ and ionized helium Zanstra temperature is $106000 \mathrm{~K}$ (Monteiro et al. 2004). Judging from these temperatures it would appear that the central star of NGC 6369 is somewhat hotter than that of NGC 3242. Yet as we shall see, the HeII lines are considerably stronger in NGC 3242. This will be discussed further in Sect. 6 on the basis of the excitation of other elements found in the nebulae.

The purpose of this paper is to study the element abundances in these nebula with the help of mid-infrared spectra, in the hope that the chemical abundances will shed some light on the evolution of these nebulae. In recent years abundances in NGC 3242 have been studied by Tsamis et al. (2003), by Barker (1985), by Henry et al. (2000), by Aller \& Czyzak (1979) and by Krabbe \& Copetti (2006). For NGC 6369 abundances have been studied by Aller \& Keyes (1987), Pena et al. (2001) and Monteiro et al. (2004). All of these groups use optical nebular spectra (taken by themselves) and in the case of NGC 3242 ultraviolet IUE spectra were also used. For NGC 6369 ultraviolet IUE spectra were taken but because of the very high extinction in the direction of this nebula they are underexposed and unusable. Barker (1985) has measurements of NGC 3242 taken at five different positions in the nebula. He uses low dispersion IUE measurements taken with the small aperture ( $3^{\prime \prime}$ diameter) which are very noisy. Henry et al. (2000) use low dispersion IUE measurements taken with the large aperture $\left(10^{\prime \prime} \times 23^{\prime \prime}\right)$. 
We have measured the spectrum of both NGC 3242 and NGC 6369 in the mid-infrared with the IRS spectrograph of the Spitzer Space Telescope (Werner et al. 2004) and with the ISO spectrograph. The use of the mid-infrared spectrum permits a more accurate determination of the abundances. The reasons for this have been discussed in earlier studies (e.g. see Pottasch \& Beintema 1999; Pottasch et al. 2000, 2001; Bernard Salas et al. 2001), and can be summarized as follows:

1) the intensity of the infrared lines is not very sensitive to the electron temperature nor to possible extinction effects;

2) use of the infrared line intensities enable a more accurate determination of the electron temperature for use with the visual and ultraviolet lines;

3) the number of observed ionization stages is doubled.

This paper is structured as follows. First the Spitzer and ISO spectra of both nebulae are presented and discussed (in Sect. 2). Then the intrinsic $\mathrm{H} \beta$ flux is determined using both the measurements of the infrared hydrogen lines and the radio continuum flux density (Sect. 3). The visible spectrum of the nebula is presented in Sect. 4 together with a new reduction of the ultraviolet (IUE) spectrum of NGC 3242 This is followed by a discussion of the nebular electron temperature and density and the chemical composition of both nebulae (Sect. 4). A comparison of the resulting abundances with those in the literature is given in Sect. 5 . In Sect. 6 a possible evolution of these nebulae is compared to other PNe. In Sect. 7 the central star is discussed especially in relation to the nebular spectrum. In Sect. 8 a general discussion and concluding remarks are given.

\section{The infrared spectrum}

Observations of both NGC 3242 and NGC 6369 were made using the Infrared Spectrograph (IRS, Houck et al. 2004) on board the Spitzer Space Telescope with AORkeys of 16463360 and 4905216 respectively. NGC 6369 was observed in staring mode using the SH module $(9.5-19.5 \mu \mathrm{m}, R \sim 600)$. In this observing mode the spectrum is taken in two positions at $1 / 3$ and $2 / 3$ of the length of the slit which are referred as the nod positions. The reduction in this case started from the droop images which are equivalent to the most commonly used Basic Calibrated Data $(b c d)$ images but lack stray-cross removal and flatfield. NGC 3242 was observed in cluster mode at 3 positions, one centered at the target and two for background of which only the closer sky position relative the target was used, with the SL, $\mathrm{SH}$, and LH modules (5.4-37 $\mu \mathrm{m})$. In cluster mode the observations are taken in the center of the slit, as opposed to the nod positions. Because the stars used for calibration were observed at the nod positions in this case the reduction started from the $b c d$ images. The data were processed using the s15.3 version of the pipeline and using a script version of Smart (Higdon et al. 2004). The tool irsclean was used to remove rogue pixels. The different cycles for a given module were combined to enhance the $\mathrm{S} / \mathrm{N}$. At this point the background images were subtracted to remove the sky contribution in NGC 3242. We note that since we are interested in line fluxes the removal of background is irrelevant for our analysis except for aiding in the removal of any rogue pixel that may have been left out by the irsclean tool (i.e. those with low flux). Then the resulting HR images were extracted using full aperture measurements and the SL module in NGC 3242 using a fix column extraction (10 pixels).

The great advantage of the IRS spectra compared to the ISO SWS spectra is the very high sensitivity of the IRS.
Otherwise the two instruments are comparable. The IRS high resolution spectra have a spectral resolution of about 600 , which is a factor of between 2 and 5 less than the resolution of the ISO SWS spectra.

\subsection{Diaphragm sizes}

The mid-infrared measurements are made with several different diaphragm sizes. Because most of the diaphragms are smaller than the size of the nebulae we are presently studying, we first discuss how the different spectra are placed on a common scale.

The spectra made with the IRS are taken with three different diaphragms: the IRS high resolution instrument (spectral resolution of about 600) measures in two spectral ranges with two different modules: the short high module $(\mathrm{SH})$ measures from $9.9 \mu \mathrm{m}$ to $19.6 \mu \mathrm{m}$ and the long high module (LH) from $18.7 \mu \mathrm{m}$ to $37.2 \mu \mathrm{m}$. The $\mathrm{SH}$ has a diaphragm size of $4.7^{\prime \prime} \times 11.3^{\prime \prime}$, while the LH is $11.1^{\prime \prime} \times 22.3^{\prime \prime}$. If the nebulae are uniformly illuminating then the ratio of the intensities would simply be the ratio of the areas measured by the two diaphragms. Since this is probably not so (because of low intensity holes in the nebulae), we may use the ratio of the continuum intensity in the region of wavelength overlap at $19 \mu \mathrm{m}$. There are other ways of determining this ratio which will be discussed presently. The SL (low resolution) spectra are made with a long slit which is 4 " wide and extends over the entire nebula.

The ISO diaphragms are somewhat larger. The SWS measurements below $12 \mu \mathrm{m}$ are made with a diaphragm $14^{\prime \prime} \times 20^{\prime \prime}$; between $12 \mu \mathrm{m}$ and $27 \mu \mathrm{m}$ it is somewhat larger $\left(14^{\prime \prime} \times 27^{\prime \prime}\right)$ and above $27 \mu \mathrm{m}$ it is $20^{\prime \prime} \times 33^{\prime \prime}$. The ISO LWS spectra (which cover a spectral region from $45 \mu \mathrm{m}$ to almost $200 \mu \mathrm{m}$ ) are taken with a diaphragm which has a diameter of about $80^{\prime \prime}$ and covers the entire nebula.

\subsubsection{NGC 3242}

The IRS measurement of NGC 3242 was centered at RA(2000) $10^{\mathrm{h}} 24^{\mathrm{m}} 46.11^{\mathrm{s}}$ and $\operatorname{Dec}(2000)-18^{\circ} 38^{\prime} 32.6^{\prime \prime}$. This is almost the same as the value measured by Kerber et al. (2003) of RA(2000) $10^{\mathrm{h}} 24^{\mathrm{m}} 46.138^{\mathrm{s}}$ and $\operatorname{Dec}(2000)-18^{\circ} 38^{\prime} 32.26^{\prime \prime}$, which is presumably the coordinate of the central star. Thus the IRS measurement was well centered on the nebula and both the LH and $\mathrm{SH}$ diaphragms measured the inner, brighter nebula. Since the LH diaphragm is larger, more of the nebula is seen in this diaphragm and therefore a correction must be made to bring the two measurements to the same scale. This was done first by making use of the fact that the two spectrographs had a small wavelength region in common at about $19 \mu \mathrm{m}$. To make the continuum emission at this wavelength equal, the $\mathrm{SH}$ emission had to be increased by 3.96 . The ratio can also be determined by using the ratio of lines of a given ion which are observed both with the SH and LH diaphragms and at the same time are also insensitive to the electron temperature and density. Such a pair of lines is from [Cl IV] at 11.76 and $20.31 \mu \mathrm{m}$, which give about the same factor between the $\mathrm{SH}$ and $\mathrm{LH}$ intensities. Normally the [Ne III] lines could also be used to obtain this ratio but this was not used in this case because the intensity of the [Ne III] line at $36.01 \mu \mathrm{m}$ is badly determined.

The measured emission line intensities for NGC 3242 are given in Table 1, after correcting the $\mathrm{SH}$ measurements by the factor 3.96 in the column labeled "intensity". The fluxes were measured using the Gaussian line-fitting routine. The last column gives the ratio of the intensity to $\mathrm{H} \beta$ where the $\mathrm{H} \beta$ is found 
Table 1. IRS spectrum of NGC 3242. The measured line intensity is given in Col. 3 . The last column gives the ratio of the line intensity to $\mathrm{H} \beta(=100)$.

\begin{tabular}{|c|c|c|c|}
\hline Identification & $\lambda(\mu \mathrm{m})$ & Intensity $^{\dagger}$ & $I / \mathrm{H} \beta$ \\
\hline [Ar III] & 8.992 & $904 \pm 30$ & 8.48 \\
\hline [S IV ] & 10.511 & $8220 \pm 160$ & 70.3 \\
\hline $\mathrm{HI}(9-7)$ & 11.306 & $38.4 \pm 2.3$ & 0.22 \\
\hline [Cl IV] & 11.760 & $70.8 \pm 3.5$ & 0.616 \\
\hline H I $(7-6+11-8)$ & 12.370 & $112.1 \pm 4.6$ & \\
\hline$[\mathrm{Ne}$ II $]$ & 12.810 & $150 \pm 3.9$ & 1.31 \\
\hline [Arv] & 13.099 & $81.5 \pm 4.7$ & 0.710 \\
\hline$[\mathrm{Ne} \mathrm{V}]$ & 14.319 & $35.6 \pm 1.6$ & 0.31 \\
\hline$[\mathrm{Ne}$ III $]$ & 15.553 & $13320 \pm 216$ & 116.0 \\
\hline H I (10-8) & 16.203 & $14.2 \pm 3.4$ & 0.15 \\
\hline $\mathrm{H}_{2}$ & 16.881 & $17.3 \pm 9.7$ & \\
\hline $\mathrm{H}_{2}$ & 16.976 & $5.2 \pm 3.3$ & \\
\hline [P III] & 17.890 & $37.0 \pm 2.0$ & 0.322 \\
\hline [S III] & 18.711 & $957 \pm 22$ & 8.33 \\
\hline H I (8-7) & 19.065 & $43.0 \pm 5.0$ & 0.401 \\
\hline [Cl IV $]$ & 20.312 & $57.0 \pm 2.66$ & 0.497 \\
\hline [Ar III] & 21.818 & $39.6 \pm 6.4$ & 0.345 \\
\hline [O IV] & 25.884 & $15724 \pm 278$ & 137 \\
\hline [S III] & 33.473 & $363: \pm 22$ & 3.16: \\
\hline [Ne III] & 36.014 & $785: \pm 98$ & 6.84: \\
\hline [O III] & 51.8 & $34700 \pm 1400$ & 162 \\
\hline$[\mathrm{N}$ III $]$ & 57.3 & $3100 \pm 140$ & 14.5 \\
\hline [O III] & 88.4 & $15500 \pm 430$ & 72.4 \\
\hline
\end{tabular}

$\dagger$ Intensities measured in units of $10^{-14} \mathrm{erg} \mathrm{cm}^{-2} \mathrm{~s}^{-1}$. The intensities below $19 \mu \mathrm{m}$ have been increased by a factor of 3.96 to bring them on the same scale as the LH intensities measured through a larger diaphragm. The intensities below $10 \mu \mathrm{m}$ are measured with the low resolution instrument.

: Indicates an uncertain value.

The last three measurements are taken from ISO.

from the strongest hydrogen line(s) measured in the IRS spectrum. It has been assumed that the ratio of the sum of the two hydrogen lines ( $n=7-6$ and $n=11-8$ ) at $12.372 \mu \mathrm{m}$ to $\mathrm{H} \beta$ has a value of $10.15 \times 10^{-3}$, which is given by Hummer \& Storey (1987) for an electron temperature of 12500 K. No correction for extinction is made since it is very small. Other hydrogen lines listed in the table can be used as well but the line at $11.306 \mu \mathrm{m}$ is blended with the He II transition $n=18-14$. All the lines predicted a value of $\mathrm{H} \beta$ through the $\mathrm{SH}$ diaphragm of $2.90 \times 10^{-11} \mathrm{erg} \mathrm{cm}^{-2} \mathrm{~s}^{-1}$ which translates to a value of $1.148 \times 10^{-10} \mathrm{erg} \mathrm{cm}^{-2} \mathrm{~s}^{-1}$ in the LH diaphragm. The [Ar III] line at $8.992 \mu \mathrm{m}$ was measured with the low resolution spectrograph (SL) using a diaphragm of $18^{\prime \prime} \times 3.6^{\prime \prime}$. The intensity has been normalized so that the other four lines measured in SL have the same intensities as these lines in $\mathrm{SH}$.

There are no ISO SWS measurements of NGC 3242 because it was not often visible to the satellite. There is however one ISO LWS measurement and the resulting line intensities are listed in the last three lines of Table 1 (taken from Liu et al. 2001). Because the LWS had a diaphragm of almost $80^{\prime \prime}$, the intensities given are for the entire nebula. To obtain the ratio of the intensities to $\mathrm{H} \beta$ (the last column) the intensities were divided by the value of $\mathrm{H} \beta$ obtained from the $6 \mathrm{~cm}$ radio continuum emission given in the next section.
Table 2. IRS spectrum of NGC 6369. The measured line intensity is given in Col. 3 . The last column gives the ratio of the line intensity to $\mathrm{H} \beta(=100)$.

\begin{tabular}{|c|c|c|c|}
\hline Identification & $\lambda(\mu \mathrm{m})$ & Intensity $^{\dagger}$ & $I / \mathrm{H} \beta$ \\
\hline [S IV] & 10.505 & $2110 \pm 66$ & 65.2 \\
\hline Н І(9-7) & 11.301 & $14.2 \pm 0.6$ & \\
\hline [Cl IV] & 11.755 & $9.95 \pm 0.7$ & 0.308 \\
\hline Н І $(7-6+11-8)$ & 12.365 & $31.3 \pm 1.3$ & \\
\hline$[\mathrm{Ne} \mathrm{II}]$ & 12.806 & $147 \pm 6.0$ & 4.14 \\
\hline [Arv] & 13.10 & $\leq 0.90$ & $\leq 0.026$ \\
\hline$[\mathrm{Mg} \mathrm{V}]$ & 13.52 & $\leq 1.27$ & $\leq 0.035$ \\
\hline [Cl II $]$ & 14.356 & $2.13 \pm 0.41$ & 0.060 \\
\hline [Ne III] & 15.546 & $4971 \pm 147$ & 139.0 \\
\hline H I (10-8) & 16.198 & $4.29 \pm 0.24$ & \\
\hline $\mathrm{H}_{2}$ & 17.036 & $2.63 \pm 0.15$ & \\
\hline [P III] & 17.882 & $28.5 \pm 0.9$ & 0.814 \\
\hline [S III] & 18.703 & $870 \pm 24$ & 24.8 \\
\hline H I (8-7) & 19.056 & $11.1 \pm 0.27$ & \\
\hline
\end{tabular}

$\dagger$ Intensities measured in units of $10^{-14} \mathrm{erg} \mathrm{cm}^{-2} \mathrm{~s}^{-1}$ and are as measured through the SH diaphragm.

: Indicates an uncertain value.

\subsubsection{NGC 6369}

The IRS measurement of NGC 6369 was centered at RA(2000) $17^{\mathrm{h}} 29^{\mathrm{m}} 20.78^{\mathrm{s}}$ and $\operatorname{Dec}(2000)-23^{\circ} 45^{\prime} 32.3^{\prime \prime}$. This is almost the same as the value measured by Kerber et al. (2003) of RA(2000) $17^{\mathrm{h}} 29^{\mathrm{m}} 20.443^{\mathrm{s}}$ and $\operatorname{Dec}(2000)-23^{\circ} 45^{\prime} 34.2^{\prime \prime}$, which again is presumably the coordinate of the central star. The IRS measurement was again well centered on the nebula and the SH diaphragm measured the inner, brighter nebula and part of the less bright hole. We do not have measurements made with the LH diaphragm. We do however have ISO measurements of this nebula. They were centered at almost exactly the same position as the IRS measurements. The IRS measurements are listed in Table 2 which is arranged in a similar way as Table 1 . The intensities listed in Col. 3 are those measured with the SH diaphragm. The ratio of the intensity to $\mathrm{H} \beta$ shown in the last column is found in the following way. The $\mathrm{H} \beta$ used is derived from the hydrogen lines listed in the table which are measured through the same diaphragm. They are interpreted in terms of $\mathrm{H} \beta$ using the theoretical ratios given by Hummer \& Storey (1987) for an electron temperature of $12500 \mathrm{~K}$ (see Sect. 4). Furthermore because the extinction is high in this nebula a correction for this is also made in this column. The correction is small in the infrared.

The ISO measurements of NGC 6369 are shown in Table 3. As can be seen from the table, the wavelength covered is considerably large than the IRS measurements but because the sensitivity is lower only the stronger lines are seen. The intensities measured are given in Col. 3 of the table and are accurate to about $20 \%$. Because the extinction, which is given in the next section, is large, the intensities corrected for extinction are given in Col. 4. In the last column the ratio of the corrected intensity to the value of $\mathrm{H} \beta$ which would have been measured through the same diaphragm (and corrected for extinction). A few words must be said about the values of $\mathrm{H} \beta$ used because it varies with the diaphragm used. The value of $\mathrm{H} \beta$ used below $12 \mu \mathrm{m}$ is found from the Brackett $\alpha$ and $\beta$ lines. Using an electron temperature of $T=12500 \mathrm{~K}$ this predicts a value of $\mathrm{H} \beta=1.53 \times 10^{-10} \mathrm{erg} \mathrm{cm}^{-2} \mathrm{~s}^{-1}$ in this region. For the spectral regions above $12 \mu \mathrm{m}$ we have increased the $\mathrm{H} \beta$ flux by the ratio of the diaphragm size; thus from $12 \mu \mathrm{m}$ to $27 \mu \mathrm{m}$ we find 
Table 3. ISO spectrum of NGC 6369. The measured line intensity is given in Col. 3 and is corrected for extinction in Col. 4. The last column gives the ratio of the line intensity to $\mathrm{H} \beta(=100)$.

\begin{tabular}{lrccc}
\hline \hline Identification & $\lambda(\mu \mathrm{m})$ & Intensity $^{\dagger}$ & Corr. Intens. & $I / \mathrm{H} \beta$ \\
\hline $\mathrm{H} \mathrm{I}(6-4)$ & 2.625 & 519 & 649 & \\
$?$ & 2.846 & 341 & 426 & 2.07 \\
$\mathrm{H} \mathrm{I}(5.4)$ & 4.051 & 978 & 1086 & \\
{$[\mathrm{Ar}$ III $]$} & 8.988 & 1940 & 2390 & 15.6 \\
{$[\mathrm{~S} \mathrm{IV}]$} & 10.507 & 11570 & 13800 & 90.2 \\
{$[\mathrm{Ne}$ II $]$} & 12.806 & 763 & 835 & 4.05 \\
{$[\mathrm{Ne} \mathrm{III}]$} & 15.549 & 27800 & 30000 & 145.6 \\
{$[\mathrm{~S} \mathrm{III}]$} & 18.705 & 5350 & 5890 & 28.6 \\
{$[\mathrm{O}$ IV $]$} & 25.884 & 2045 & 2390 & 10.6 \\
{$[\mathrm{~S} \mathrm{III}]$} & 33.469 & 4060 & 4220 & 11.7 \\
{$[\mathrm{Ne}$ III $]$} & 35.992 & 4820 & 4980 & 13.8 \\
{$[\mathrm{O} \mathrm{III}]$} & 51.822 & 68600 & & 111.4 \\
{$[\mathrm{~N} \mathrm{III}]$} & 57.306 & 10400 & & 16.9 \\
{$[\mathrm{O}$ I $]$} & 63.163 & 5850 & & 9.5 \\
{$[\mathrm{O}$ III $]$} & 88.351 & 21360 & & 34.7 \\
{$[\mathrm{~N} \mathrm{III}]$} & 121.905 & 201 & & 0.33 \\
{$[\mathrm{O}$ I $]$} & 145.486 & 155 & & 0.25 \\
{$[\mathrm{C} \mathrm{II}]$} & 157.712 & 674 & & 1.09 \\
\hline
\end{tabular}

${ }^{\dagger}$ Intensities measured in units of $10^{-14} \mathrm{erg} \mathrm{cm}^{-2} \mathrm{~s}^{-1}$ and have been corrected for extinction in Col. 4. The determination of $I / \mathrm{H} \beta$ is discussed in the text.

: Indicates an uncertain value.

$2.06 \times 10^{-10} \mathrm{erg} \mathrm{cm}^{-2} \mathrm{~s}^{-1}$ and above this wavelength (and less than $40 \mu \mathrm{m}$ ) we use $3.60 \times 10^{-10} \mathrm{erg} \mathrm{cm}^{-2} \mathrm{~s}^{-1}$. This is the correct procedure as long as the nebula is larger than the largest diaphragm used and the emission is uniform. This may be checked by comparing the continuum emission ratios measured at the transition wavelengths. This gives a consistent result although the continuum is rather noisy, especially at $12 \mu \mathrm{m}$. Another manner of check is to note that the ratio of the [Ne III] lines 15.5/36.0, which has only a very small dependence on electron temperature and density, has its predicted theoretical value. For the wavelength region above $50 \mu \mathrm{m}$ the diaphragm is large enough to include the entire nebula; therefore The value of $\mathrm{H} \beta$ used is that found from the $6 \mathrm{~cm}$ flux density: $6.16 \times 10^{-10} \mathrm{erg} \mathrm{cm}^{-2} \mathrm{~s}^{-1}$ (see the following section).

The values of $\mathrm{I} / \mathrm{H} \beta$ found from the IRS and the ISO measurements can be compared for four lines in common to the two instruments (see Tables 2 and 3). In three of the four cases agreement is within $10 \%$. Only for the [S IV] line is the agreement less good. We suggest that the difficulty lies with the IRS measurement because this part of the spectrum is badly calibrated. The line measured in different orders differs by about $34 \%$. In the discussion below the ISO value will be used for this line and the IRS values for the other lines.

\section{Extinction}

The extinction may be found both by a comparison of radio emission with $\mathrm{H} \beta$ flux and by a comparison of observed and theoretical Balmer decrement, both of which we will discuss.

\subsection{The $6 \mathrm{~cm}$ radio emission}

The $6 \mathrm{~cm}$ flux density for both nebulae has been measured by Griffith et al. (1994) in the Parkes-MIT-NRAO survey. Their reduction gave two values: one using a Fixed width fit and the other a General width fit. For NGC 6369 they find 2041 and $1893 \mathrm{mJy}$ and for NGC 3242760 and $731 \mathrm{mJy}$. In addition NGC 6369 was measured by Milne \& Aller (1975) who find a value of $2002 \mathrm{mJy}$ for this object. We will use a value of $2000 \mathrm{mJy}$ for NGC 6369 which, using values of $T_{\mathrm{e}}$ and helium abundance determined below together with the equation quoted in Pottasch (1984), implies a total $\mathrm{H} \beta$ flux of $6.16 \times$ $10^{-10} \mathrm{erg} \mathrm{cm}^{-2} \mathrm{~s}^{-1}$ for this object. Similarly a value of $745 \mathrm{mJy}$ for NGC 3242 implies a total $\mathrm{H} \beta$ flux of $2.14 \times 10^{-10} \mathrm{erg} \mathrm{cm}^{-2} \mathrm{~s}^{-1}$ for this object.

\subsection{Extinction}

The measured value of the integrated $\mathrm{H} \beta$ flux for NGC 6369 and NGC 3242 is $4.76 \times 10^{-12}$ and $1.62 \times 10^{-11} \mathrm{erg} \mathrm{cm}^{-2} \mathrm{~s}^{-1}$ respectively (see Acker et al. 1992). Using the value of $\mathrm{H} \beta$ from the radio measurements leads to a value of $C=2.116$ or $E_{B-V}=1.444$. For NGC 3242 these values are $C=0.12$ $E_{B-V}=0.083$.

The extinction determined from the Balmer decrement differs somewhat according to different authors. For NGC 6369 Monteiro et al. (2004) find $C=2.17$, Aller \& Keyes (1987) give $C=2.23$ and Pena et al. (2001) find $C=1.9$. For NGC 3242 Henry et al. (2000) give $C=0$, Aller \& Czyzak (1979) give $C=0.14$, and Barker (1985) finds $C=0.15$. These values are in rough agreement with those found from the radio/ $\mathrm{H} \beta$ method. Since this method is the most accurate determination, this value will be used when necessary in this paper.

\subsection{The visual spectrum}

The visual spectrum has been measured by several authors for each of the nebulae. We list here the results of three of the most recent high resolution spectra for each nebula. The line intensities reported have been corrected by each author for a value of extinction determined by them to obtain a theoretically correct Balmer decrement. The result are listed in Tables 4 and 5, where the last column lists the average value which we have used. No attempt has been made to use a common extinction correction because then the Balmer decrement will be incorrect. The value of extinction $C$ which the individual authors found is listed at the bottom of the table. All authors estimate that the strongest lines have a $10 \%$ error, the intermediate strength lines (about $5 \%$ of $\mathrm{H} \beta$ ) have about $20 \%$ error and the weakest lines have about $30 \%$ error. The average intensities have about the same error.

The measured optical line fluxes of NGC 3242 are shown in Table 4 and that of NGC 6369 in Table 5.

\subsection{The IUE ultraviolet spectrum}

Only a few IUE observations of NGC 6369 were made. All of them were underexposed in spite of the fact that the nebula is so bright. The very large extinction reduces the ultraviolet emission in this case to such an extent that the measurements are not useful. By contrast there are 91 low resolution IUE observations of NGC 3242 as well as six high resolution observations of this nebula. Seventy of the low resolution measurements were taken with the large aperture $\left(10^{\prime \prime} \times 23^{\prime \prime}\right)$ with varying exposure times, and 12 were taken with a small aperture ( $3^{\prime \prime}$ diameter). We find that the small aperture measurements are too noisy and we do not use them. The large aperture measurements do not cover the entire nebula. We have used two of the highest $\mathrm{S} / \mathrm{N}$ observations 
Table 4. Visual spectrum of NGC 3242.

\begin{tabular}{|c|c|c|c|c|c|}
\hline \multirow{2}{*}{$\begin{array}{c}\lambda \\
(\AA)\end{array}$} & \multirow[t]{2}{*}{ Ion } & \multicolumn{3}{|c|}{ Intensities $^{\dagger}$} & \multirow{2}{*}{$\begin{array}{l}\text { Average } \\
\text { Intens. }\end{array}$} \\
\hline & & (1) & (2) & (3) & \\
\hline 3426 & {$[\mathrm{Ne} \mathrm{V}]$} & & 1.73 & & 1.73 \\
\hline $3727^{*}$ & [O II] & 14.4 & 6.0 & 11.2 & 12 \\
\hline 3869 & [Ne III] & 103 & 88.8 & 91 & 93 \\
\hline 4101 & $\mathrm{H} \delta$ & 26.6 & 27.0 & 25.8 & \\
\hline 4267 & C II & 0.62 & 0.77 & 0.72 & 0.70 \\
\hline 4340 & $\mathrm{H} \gamma$ & 47.4 & 46.8 & 44.6 & \\
\hline 4363 & [O III] & 13.5 & 14.0 & 12.0 & 13.5 \\
\hline 4471 & $\mathrm{He} \mathrm{I}$ & 4.12 & 3.52 & 3.56 & 3.7 \\
\hline 4686 & He II & 25.7 & 40.5 & 23.0 & 28 \\
\hline 4711 & [Ar IV] & 4.89 & 5.95 & 4.0 & 4.9 \\
\hline 4724 & [Ne IV] & 0.0493 & & & 0.049 \\
\hline 4725 & [Ne IV] & 0.0407 & & & 0.041 \\
\hline 4740 & [Ar IV] & 4.52 & 5.29 & 3.58 & 4.5 \\
\hline 4861 & $\mathrm{H} \beta$ & 100 & 100 & 100 & 100 \\
\hline 5007 & [O III] & 1280 & 1205 & 1260 & 1260 \\
\hline 5517 & [Cl III] & 0.317 & 0.26 & & 0.30 \\
\hline 5538 & [Cl III] & 0.277 & 0.25 & & 0.27 \\
\hline 5755 & [N II] & 0.0634 & & 0.065 & 0.064 \\
\hline 5876 & $\mathrm{He} \mathrm{I}$ & 11.6 & 10.3 & 9.2 & 11.0 \\
\hline 6312 & [S III] & 0.631 & 0.53 & 0.53 & 0.56 \\
\hline 6563 & $\mathrm{H} \alpha$ & 285 & & 282 & \\
\hline 6584 & [N II] & 2.63 & 1.51 & 3.48 & 2.6 \\
\hline 6717 & [S II] & 0.278 & 0.24 & & 0.27 \\
\hline 6731 & [S II $]$ & 0.392 & 0.26 & & 0.39 \\
\hline 7005 & [Ar V] & 0.0835 & & 0.063 & 0.080 \\
\hline 7135 & [Ar III] & 6.99 & & 6.92 & 6.96 \\
\hline 7263 & [Ar IV] & 0.124 & & 0.11 & 0.19 : \\
\hline 8045 & [Cl IV] & & & 0.41 & 0.41 \\
\hline C & & 0.10 & 0.10 & 0.14 & \\
\hline
\end{tabular}

$\dagger$ References; (1) Tsamis et al. (2003); (2) Krabbe et al. (2006); (3) Aller \& Czyzak (1979).

: Indicates uncertain values.

* This is a blend of $\lambda 3726$ and $\lambda 3729$ lines.

$C$ is the extinction.

made with long exposure times both in the short and the long wavelength regions. These are SWP15495 and SWP16418 for the short wavelength region and LWR11973 and LWR 12678 for the long wavelength region. We have checked that the strongest lines are not saturated by measuring these same lines on spectra with shorter exposure times: SWP15495, SWP 16419 and LWR12679. In addition two high resolution large aperture measurements are used: SWP03643 and LWR03206. All of these measurements are centered within $6 "$ of the center of the nebula and thus are representative of the nebula as a whole. The results are shown in Table 6. The uncertainties are about the same as given by Henry et al. (2000): about $10 \%$ for the strongest lines and about $25 \%$ for the weaker lines. From the high resolution spectra we find the C III ratio $1906 / 1909=1.4 \pm 0.1$ and the Ne IV ratio 2422/2425 $=0.92 \pm 0.5$.

The extinction correction was made by assuming a theoretical ratio for the He II line ratio $\lambda 1640 / \lambda 4686 \AA$ at $T=11500 \mathrm{~K}$ and an $N_{\mathrm{e}}$ of $10^{4} \mathrm{~cm}^{-3}$. The ratio of $\lambda 1640$ to $\mathrm{H} \beta$ can then be found using the $\lambda 4686 / \mathrm{H} \beta$ ratio in Table 4 . A further correction for extinction relative to $\lambda 1640 \AA$ is then made using the reddening curve of Fluks et al. (1994) but because of the small extinction this is never more than $20 \%$, which indicates the
Table 5. Visual spectrum of NGC 6369.

\begin{tabular}{|c|c|c|c|c|c|}
\hline \multirow{2}{*}{$\begin{array}{c}\lambda \\
(\AA)\end{array}$} & \multirow[t]{2}{*}{ Ion } & \multicolumn{3}{|c|}{ Intensities $^{\dagger}$} & \multirow{2}{*}{$\begin{array}{l}\text { Average } \\
\text { Intens. }\end{array}$} \\
\hline & & $(1)$ & $(2)$ & (3) & \\
\hline 3726 & [O II] & & & 63 & 63 \\
\hline 3729 & [O II] & & & 39 & 39 \\
\hline 3869 & [Ne III] & & 84.2 & 89.0 & 88 \\
\hline 4340 & $\mathrm{H} \gamma$ & & 46.4 & & \\
\hline 4363 & [O III] & & 14.2 & 8.3 & 11 \\
\hline 4686 & He II & 1.4 & 2.4 & 10.0 & 2.5 \\
\hline 4711 & [Ar IV] & & & 1.3 & 1.3 \\
\hline 4740 & [Ar IV] & & & 1.5 & 1.5 \\
\hline 4861 & $\mathrm{H} \beta$ & 100 & 100 & 100 & 100 \\
\hline 5007 & [O III] & 1241 & 1307 & 1260 & 1260 \\
\hline 5517 & [Cl III] & 1.0 & 0.48 & & 0.6 \\
\hline 5538 & [Cl IIII] & 1.0 & 0.91 & & 0.96 \\
\hline 5755 & {$[\mathrm{~N} \mathrm{II}]$} & 2.0 & 1.77 & 1.9 & 1.9 \\
\hline 5876 & He I & 16 & 15.2 & 15 & 15.5 \\
\hline 6312 & [S III $]$ & 1.4 & 1.3 & 7.0 & 1.4 \\
\hline 6563 & $\mathrm{H} \alpha$ & 287 & 285 & & \\
\hline 6584 & {$[\mathrm{~N} \mathrm{II}]$} & 73 & 62.2 & 87.2 & 73 \\
\hline 6717 & [S II $]$ & 4.4 & 2.81 & 4.4 & 4.2 \\
\hline 6731 & [S II] & 6.3 & 5.25 & 7.1 & 6.8 \\
\hline 7135 & [Ar III] & & 16.6 & & 16.6 \\
\hline
\end{tabular}

$\dagger$ References: (1) Monteiro et al. (2004); (2) Aller \& Keyes (1987);

(3) Pena et al. (2001).

: Indicates uncertain values.

* This is a blend of $\lambda 3726$ and $\lambda 3729$ lines.

Table 6. IUE spectrum of NGC 3242.

\begin{tabular}{clccc}
\hline \hline$\lambda$ & Ion & \multicolumn{3}{c}{ Intensities } \\
\cline { 3 - 5 }$(\AA)$ & & $(1)$ & $(2)$ & $(I / \mathrm{H} \beta)$ \\
\hline 1239 & N V & 19 & 1.32 & 7.4 \\
1335 & C II & 16 & 1.0 & 4.76 \\
1400 & O IV & 7.8 & 4.68 & 2.19 \\
1485 & N IV] & 26 & 15.6 & 6.44 \\
1548 & C IV & 110 & 66.0 & 30.8 \\
1575 & {$[\mathrm{Ne}$ V] } & 12 & 7.2 & 3.36 \\
1640 & He II & 622 & 373 & 174 \\
1663 & O III] & 45 & 27 & 12.6 \\
1750 & N III] & 15.8 & 9.48 & 4.43 \\
1909 & C III] & 675 & 405 & 189 \\
2298 & C III] & 40 & 27 & 12.6 \\
2325 & C II] & 26.8 & 17.0 & 7.95 \\
2423 & {$[\mathrm{Ne}$ IV] } & 44 & 30.1 & 14.1 \\
2512 & He II & 17 & 8.67 & 4.05 \\
2733 & He II & 27 & 11.4 & 5.32 \\
2837 & {$[$ Fe IV] } & 45 & 22.2 & 10.4 \\
3024 & O III & 20 & 7.8 & 3.64 \\
3048 & O III & 50 & 19.8 & 9.24 \\
3133 & O III & 100 & 39.0 & 18.2 \\
3103 & He II & 38 & 13.8 & 6.44 \\
\hline
\end{tabular}

(1) Measured intensity from low resolution spectra in units of $10^{-13} \mathrm{erg} \mathrm{cm}^{-2} \mathrm{~s}^{-1}$; (2) Intensity corrected for diaphragm size and extinction in units of $10^{-12} \mathrm{erg} \mathrm{cm}^{-2} \mathrm{~s}^{-1} . I / \mathrm{H} \beta$ is normalized to $\mathrm{H} \beta=100$.

uncertainties of the UV intensities above the errors of measurement given above. The results are shown in the last two columns of Table 6. 
Table 7. Electron density indicators in NGC 3242.

\begin{tabular}{lcccc}
\hline \hline Ion & $\begin{array}{c}\text { Ioniz. } \\
\text { Pot. }(\mathrm{eV})\end{array}$ & $\begin{array}{c}\text { Lines } \\
\text { used }\end{array}$ & $\begin{array}{c}\text { Observed } \\
\text { ratio }\end{array}$ & $\begin{array}{c}N_{\mathrm{e}} \\
\left(\mathrm{cm}^{-3}\right)\end{array}$ \\
\hline$[\mathrm{S} \mathrm{II}]$ & 10.4 & $6731 / 6716$ & 1.38 & 1900 \\
{$[\mathrm{O}$ II $]$} & 13.6 & $3626 / 3729$ & 1.61 & 2700 \\
{$[\mathrm{~S} \mathrm{III}]$} & 23.3 & $33.5 / 18.7$ & $0.38:$ & 2800 \\
{$[\mathrm{Cl}$ III $]$} & 23.8 & $5538 / 5518$ & 1.08 & 3000 \\
$\mathrm{C} \mathrm{III}]$ & 24.4 & $1906 / 1909$ & 1.29 & $3300:$ \\
{$[\mathrm{O}$ III $]$} & 35.1 & $51.8 / 88.4$ & 2.24 & $900:$ \\
{$[\mathrm{Ar}$ IV $]$} & 40.7 & $4711 / 4740$ & 1.08 & $2100:$ \\
{$[\mathrm{Ne}$ IV $]$} & 63.5 & $2425 / 2422$ & 0.92 & $5000:$ \\
\hline
\end{tabular}

: Indicates uncertain values.

Wavelengths of the far infrared lines are in microns, the rest are in Angstroms.

Table 8. Electron density indicators in NGC 6369.

\begin{tabular}{lcccc}
\hline \hline Ion & $\begin{array}{c}\text { Ioniz. } \\
\text { Pot. }(\mathrm{eV})\end{array}$ & $\begin{array}{c}\text { Lines } \\
\text { used }\end{array}$ & $\begin{array}{c}\text { Observed } \\
\text { ratio }\end{array}$ & $\begin{array}{c}N_{\mathrm{e}} \\
\left(\mathrm{cm}^{-3}\right)\end{array}$ \\
\hline$[\mathrm{S} \mathrm{II}]$ & 10.4 & $6731 / 6716$ & 1.51 & 2300 \\
{$[\mathrm{O}$ II $]$} & 13.6 & $3626 / 3729$ & 1.62 & 2800 \\
{$[\mathrm{~S} \mathrm{III}]$} & 23.3 & $33.5 / 18.7$ & 0.409 & 2900 \\
{$[\mathrm{Cl}$ III $]$} & 23.8 & $5538 / 5518$ & $1.3:$ & $3000:$ \\
{$[\mathrm{O}$ III $]$} & 35.1 & $51.8 / 88.4$ & 2.1 & $800:$ \\
{$[\mathrm{Ar}$ IV $]$} & 40.7 & $4711 / 4740$ & 0.87 & $4000:$ \\
\hline
\end{tabular}

: Indicates uncertain values. Wavelength units as in Table 7.

\section{Chemical composition of the nebulae}

The method of analysis is as follows. First the electron density and temperature as a function of the ionization potential are determined. The ionic abundances are then determined, using density and temperature appropriate for the ion under consideration. Then the element abundances are found for those elements in which a sufficient number of ionic abundances have been derived.

\subsection{Electron density}

The ions used to determine $N_{\mathrm{e}}$ for NGC 3242 are listed in the first column of Table 7; those for NGC 6369 are listed in Table 8. The ionization potential required to reach this stage of ionization, and the wavelengths of the lines used, are given in Cols. 2 and 3 of the tables. Note that the wavelength units are $\AA$ when 4 figures are given and microns when 3 are shown. The observed ratio of the lines is given in the fourth column; the corresponding $N_{\mathrm{e}}$ is given in the fifth column. The temperature used is discussed in the following section, but is unimportant since these line ratios are essentially determined by the density. The density from the C III] lines in NGC 3242 is uncertain because the two measurements used differed substantially..

There is no indication that the electron density varies with ionization potential in a systematic way in either of the nebulae. The electron density appears to be about $2500 \mathrm{~cm}^{-3}$ in NGC 3242 and slightly higher (about $2800 \mathrm{~cm}^{-3}$ ) in NGC 6369. The error is about $20 \%$ in NGC 3242 and slightly higher in NGC 6369. We will use these densities in further discussion of the abundances, but any value between $1500 \mathrm{~cm}^{-3}$ and $3000 \mathrm{~cm}^{-3}$ will give the same values of abundance.
Table 9. Electron temperature indicators in NGC 3242.

\begin{tabular}{lcccc}
\hline \hline Ion & $\begin{array}{c}\text { Ioniz. } \\
\text { Pot. }(\mathrm{eV})\end{array}$ & $\begin{array}{c}\text { Lines } \\
\text { used } \dagger\end{array}$ & $\begin{array}{c}\text { Observed } \\
\text { ratio }\end{array}$ & $\begin{array}{c}T_{\mathrm{e}} \\
(\mathrm{K})\end{array}$ \\
\hline$[\mathrm{N} \mathrm{II}]$ & 14.5 & $5755 / 6584$ & 0.0211 & 11000 \\
{$[\mathrm{~S} \mathrm{III}]$} & 23.3 & $6312 / 18.7$ & 0.0 .075 & 11800 \\
{$[\mathrm{Ar}$ III $]$} & 27.6 & $7136 / 21.8$ & 20.3 & 10600 \\
{$[\mathrm{O} \mathrm{III}]$} & 35.1 & $4363 / 5007$ & 0.0106 & 11780 \\
{$[\mathrm{O} \mathrm{III}]$} & 35.1 & $1663 / 5007$ & 0.0099 & 10800 \\
{$[\mathrm{O} \mathrm{III}]$} & 35.1 & $5007 / 51.8$ & 7.90 & 11000 \\
{$[\mathrm{Ne}$ III $]$} & 41.0 & $3869 / 15.5$ & 0.89 & 11000 \\
{$[\mathrm{O}$ IV $]$} & 54.9 & $1400 / 25.9$ & 0.016 & $12000:$ \\
\hline
\end{tabular}

: Indicates uncertain value.

$\dagger$ When the wavelength has 4 figures it has the units of Angstrom and 3 figures is micron.

Table 10. Electron temperature indicators in NGC 6369.

\begin{tabular}{lcccc}
\hline \hline Ion & $\begin{array}{c}\text { Ioniz. } \\
\text { Pot. }(\mathrm{eV})\end{array}$ & $\begin{array}{c}\text { Lines } \\
\text { used } \dagger\end{array}$ & $\begin{array}{c}\text { Observed } \\
\text { ratio }\end{array}$ & $\begin{array}{c}T_{\mathrm{e}} \\
(\mathrm{K})\end{array}$ \\
\hline$[\mathrm{N} \mathrm{II}]$ & 14.5 & $5755 / 6584$ & 0.022 & 11000 \\
{$[\mathrm{~S} \mathrm{III}]$} & 23.3 & $6312 / 18.7$ & 0.0 .049 & 10000 \\
{$[\mathrm{Ar}$ III $]$} & 27.6 & $7136 / 8.99$ & 1.07 & 10000 \\
{$[\mathrm{O} \mathrm{III}]$} & 35.1 & $4363 / 5007$ & 0.0096 & 11300 \\
{$[\mathrm{O}$ III $]$} & 35.1 & $5007 / 51.8$ & 11.3 & 11500 \\
{$[\mathrm{Ne}$ III $]$} & 41.0 & $3869 / 15.5$ & 0.604 & 10500 \\
\hline
\end{tabular}

: Indicates uncertain value.

$\dagger$ When the wavelength has 4 figures it has the units of Angstrom and 3 figures is micron.

\subsection{Electron temperature}

A number of ions have lines originating from energy levels far enough apart that their ratio is sensitive to the electron temperature. These are listed in Tables 9 and 10 for each of the nebulae. These tables are arranged similarly to the previous tables. No temperature gradient is seen in either of the nebulae. Both nebulae have very similar electron temperatures: the temperature of NGC 3242 is about $11500 \mathrm{~K}$ while that of NGC 6369 seems to be slightly lower: $T=11000 \mathrm{~K}$. The temperature for NGC 3242 is better determined because ultraviolet measurements are available. Although a weak line is seen at $14.3 \mu \mathrm{m}$, no temperature can be obtained from $\mathrm{Ne} \mathrm{V}$ because no good measurement of the ultraviolet line at $\lambda 3425$ has been made.

\subsection{Ion and element abundances}

The ion abundances have been determined using the following equation:

$\frac{N_{\text {ion }}}{N_{\mathrm{p}}}=\frac{I_{\text {ion }}}{I_{\mathrm{H}_{\beta}}} N_{\mathrm{e}} \frac{\lambda_{\mathrm{ul}}}{\lambda_{\mathrm{H}_{\beta}}} \frac{\alpha_{\mathrm{H}_{\beta}}}{A_{\mathrm{ul}}}\left(\frac{N_{\mathrm{u}}}{N_{\text {ion }}}\right)^{-1}$

where $I_{\mathrm{ion}} / I_{\mathrm{H}_{\beta}}$ is the measured intensity of the ionic line compared to $\mathrm{H} \beta, N_{\mathrm{p}}$ is the density of ionized hydrogen, $\lambda_{\mathrm{ul}}$ is the wavelength of the line, $\lambda_{\mathrm{H}_{\beta}}$ is the wavelength of $\mathrm{H} \beta, \alpha_{\mathrm{H}_{\beta}}$ is the effective recombination coefficient for $\mathrm{H} \beta, A_{\mathrm{ul}}$ is the Einstein spontaneous transition rate for the line, and $N_{\mathrm{u}} / N_{\text {ion }}$ is the ratio of the population of the level from which the line originates to the total population of the ion. This ratio has been determined using a five level atom. The atomic data used is given in the paper of Pottasch \& Beintema (1999). A 5 level atom is used in all cases. For iron this is justified because collisional rates to higher levels 
followed by cascade through the lower levels are quite small in comparison to direct collisions to the lower levels.

The results are given in Tables 11 and 12, where the first column lists the ion concerned, the second column the line used for the abundance determination, and the third column gives the intensity of the line used relative to $\mathrm{H} \beta=100$. The fourth column gives the value of the ionic abundance assuming the ion is formed at $T=11500 \mathrm{~K}$ for NGC 3242 and $T=11000 \mathrm{~K}$ for NGC 6396, while the fifth column gives the ionization correction factor (ICF), which has been determined empirically. Notice that the ICF is close to unity for all elements listed in the table except for $\mathrm{Fe}$ and $\mathrm{P}$.

The error of measurement of the IRS intensities as can be seen in Table 1 is usually small, often not more than 5-7\%. In the few cases when the error is large this has either been indicated with a ":" or by not using the line. The correction for adjusting the SH to LH intensity scales and the diaphragm size is also small, about $10 \%$. This includes the assumption that the unmeasured parts of the nebula have the same composition as the measured parts. This has been checked in the optical region by Barker (1985) and can be seen to be a reasonable approximation in the ultraviolet region by comparing the $I U E$ spectra obtained in different parts of the nebula. The uncertainty of the collisional strengths introduces an error of $10-15 \%$ so that the total error for the ions of neon, sulfur and argon determined with the IRS measurements is less than $20 \%$. This will also be true of the abundances of these elements because the ICF for these elements is close to unity. The error for the nitrogen and oxygen abundances is somewhat higher because the visual and ultraviolet measurements are less certain. In addition the temperature is more important for these ions and the total errors may be twice as large. The element abundances are given in the last column. The carbon recombination line abundance for NGC 3242 is given at the end of Table 11. It is somewhat more than a factor of 3 higher than the value obtained from the collisional transition. This difference is found in other PNe as well but is not yet understood. No recombination line has been measured in NGC 6369.

The helium abundance was derived using the theoretical work of Benjamin et al. (1999) and Porter et al. (2005) For recombination of singly ionized helium, most weight is given to the $\lambda 5875 \AA$ line, because the theoretical determination of this line is the most reliable.

\section{Comparison with other abundance determinations}

Tables 13 and 14 show a comparison of our abundances with three of the most important determinations in the past 20 years for each nebulae. For NGC 3242 reasonable agreement is found. Good agreement is found for oxygen; this is because the same electron temperature is used for the most important oxygen ion. For the other elements the agreement is less good. For nitrogen a somewhat higher abundance is found which is probably due to the fact that the most important ionization stages have not been measured earlier. Nitrogen is somewhat higher than in the Sun. A C/O ratio substantially lower than unity and close to the solar value is found but the error is about $30 \%$. Sulfur is in good agreement with earlier determinations but is a factor of 4 lower than the solar value. This is a general phenomenon in PNe and has been discussed earlier (e.g. Pottasch \& BernardSalas 2006; Bernard-Salas et al. 2008). Phosphorus, which has never been measured before in this nebula and is slightly uncertain because only a single ionization stage has been measured, is
Table 11. Ionic concentrations and chemical abundances in NGC 3242. Wavelengths in Angstrom for all values of $\lambda$ above 1000, otherwise in $\mu \mathrm{m}$.

\begin{tabular}{lccccc}
\hline \hline Ion & $\lambda$ & $(\mathrm{I} / \mathrm{H} \beta) \dagger$ & $N_{\text {ion }} / N_{\mathrm{p}}$ & $\mathrm{ICF}$ & $N_{\mathrm{el} .} / N_{\mathrm{p}}$ \\
\hline $\mathrm{He}^{+}$ & 5875 & 11.1 & 0.0707 & & \\
$\mathrm{He}^{++}$ & 4686 & 25 & 0.0212 & 1.0 & 0.092 \\
$\mathrm{C}^{+}$ & 2324 & 7.65 & $6.8(-6)$ & & \\
$\mathrm{C}^{++}$ & 1909 & 181 & $1.61(-4)$ & & \\
$\mathrm{C}^{+3}$ & 1548 & 29.4 & $2.6(-5)$ & 1.0 & $1.95(-4)$ \\
$\mathrm{N}^{+}$ & 6584 & 2.7 & $4.1(-7)$ & & \\
$\mathrm{N}^{++}$ & 1750 & 4.23 & $(-5)$ & & \\
$\mathrm{N}^{++}$ & 57.3 & 14.5 & $5.7(-5)$ & & \\
$\mathrm{N}^{+3}$ & 1485 & 7.0 & $4.39(-5)$ & & \\
$\mathrm{N}^{+4}$ & 1239 & $5.1:$ & $3.6(-5):$ & 1.0 & $1.35(-4)$ \\
$\mathrm{O}^{+}$ & 3729 & 5.36 & $5.1(-6)$ & & \\
$\mathrm{O}^{++}$ & 5007 & 1280 & $3.18(-4)$ & & \\
$\mathrm{O}^{++}$ & 51.7 & 162 & $4.38(-4)$ & & \\
$\mathrm{O}^{+3}$ & 25.8 & 137 & $4.3(-5)$ & 1.0 & $3.8(-4)$ \\
$\mathrm{Ne}^{+}$ & 12.8 & 1.31 & $1.8(-6)$ & & \\
$\mathrm{Ne}^{++}$ & 15.5 & 116 & $7.53(-5)$ & & \\
$\mathrm{Ne}^{++}$ & 3869 & 103 & $6.5(-5)$ & & \\
$\mathrm{Ne}^{+3}$ & 2423 & 12.6 & $1.25(-5)$ & & \\
$\mathrm{Ne}^{+4}$ & 14.3 & 0.31 & $3.1(-8)$ & 1.0 & $9.0(-5)$ \\
$\mathrm{S}^{+}$ & 6731 & 0.392 & $1.8(-8)$ & & \\
$\mathrm{S}^{++}$ & 18.7 & 8.33 & $8.38(-7)$ & & \\
$\mathrm{S}^{++}$ & 6312 & 0.61 & $8.3(-7)$ & & \\
$\mathrm{S}^{+3}$ & 10.5 & 70.3 & $1.94(-6)$ & 1.0 & $2.8(-6)$ \\
$\mathrm{Ar}^{++}$ & 8.99 & 8.48 & $8.32(-7)$ & & \\
$\mathrm{Ar}^{++}$ & 21.8 & 0.345 & $5.2(-7)$ & & \\
$\mathrm{Ar}^{++}$ & 7135 & 7.0 & $5.0(-6)$ & & \\
$\mathrm{Ar}^{+3}$ & 4740 & 4.3 & $8.49(-7)$ & & \\
$\mathrm{Ar}^{+4}$ & 7005 & 0.083 & $1.4(-8)$ & 1.0 & $1.7(-6)$ \\
$\mathrm{Cl}^{++}$ & 5538 & 0.28 & $2.7(-8)$ & & \\
$\mathrm{Cl}^{+3}$ & 11.8 & 0.616 & $3.51(-8)$ & & \\
$\mathrm{Cl}^{+3}$ & 8045 & $0.5:$ & $4.0(-8):$ & 1.1 & $7.5(-8)$ \\
$\mathrm{Fe}^{++}$ & 22.9 & 1.55 & $4.7(-7)$ & & $:$ \\
$\mathrm{Fe}^{++}$ & 33.0 & 0.51 & $5.8(-7)$ & 1.6 & $8.0(-7)$ \\
$\mathrm{P}^{++}$ & 17.9 & 0.322 & $1.33(-8):$ & $3.5:$ & $4.5(-8):$ \\
$\mathrm{C}^{++}$ & 4267 & 0.62 & $6.0(-4):$ & & \\
\hline
\end{tabular}

$\dagger$ Intensities given with respect to $\mathrm{H} \beta=100$.

: Indicates uncertain value.

considerably lower than solar. Chlorine also seems rather strongly depleted compared to the solar abundance. The solar abundance listed in the table is taken from Asplund et al. (2005). Note that for solar sulfur and chlorine more weight has been given to the abundance determination in meteorites since this determination is more accurate than for the Sun itself. Neon and argon abundances are taken from the references given in Pottasch \& Bernard-Salas (2006) and differ substantially with those given by Asplund et al. (2005). We point out that the solar abundances have changed greatly in the past 10 years and it is uncertain that there will be no further changes in the near future (see discussion by Bernard-Salas et al. 2008).

For NGC 6369 the agreement is somewhat less good. The reason for this is that the comparison determinations use only the visible spectra since no ultraviolet measurements have been made. For nitrogen only the $\mathrm{N}^{+}$abundance can be measured and a large correction for higher ionization stages must be made. Besides this the very high extinction correction which must be made in this nebula leads to larger uncertainties in line 
Table 12. Ionic concentrations and chemical abundances in NGC 6369. Wavelengths in Angstrom for all values of $\lambda$ above 1000, otherwise in $\mu \mathrm{m}$.

\begin{tabular}{lccccc}
\hline \hline Ion & $\lambda$ & $(I / \mathrm{H} \beta) \dagger$ & $N_{\text {ion }} / N_{\mathrm{p}}$ & ICF & $N_{\text {el. }} / N_{\mathrm{p}}$ \\
\hline $\mathrm{He}^{+}$ & 5875 & 15.5 & 0.100 & & \\
$\mathrm{He}^{++}$ & 4686 & 2.2 & 0.0018 & 1.0 & 0.102 \\
$\mathrm{~N}^{+}$ & 6584 & 73 & $1.17(-5)$ & & \\
$\mathrm{N}^{+}$ & 122 & 0.33 & $1.85(-5)$ & & \\
$\mathrm{N}^{++}$ & 57.3 & 16.9 & $6.57(-5)$ & 1.2 & 9.4 \\
$\mathrm{O}^{+}$ & 3729 & 39 & $4.2(-5)$ & & \\
$\mathrm{O}^{++}$ & 5007 & 1260 & $3.42(-4)$ & & \\
$\mathrm{O}^{++}$ & 51.8 & 111 & $3.0(-4)$ & & \\
$\mathrm{O}^{+3}$ & 25.8 & 10.6 & $3.2(-6)$ & 1.0 & $3.9(-4)$ \\
$\mathrm{Ne}^{+}$ & 12.8 & 4.1 & $6.25(-6)$ & & \\
$\mathrm{Ne}^{++}$ & 15.5 & 142 & $8.97(-5)$ & & \\
$\mathrm{Ne}^{++}$ & 3869 & 88 & $5.29(-5)$ & 1.0 & $9.7(-5)$ \\
$\mathrm{S}^{+}$ & 6731 & 6.8 & $3.22(-7)$ & & \\
$\mathrm{S}^{++}$ & 18.7 & 28.6 & $2.83(-6)$ & & \\
$\mathrm{S}^{++}$ & 6312 & 1.4 & $2.13(-6)$ & & \\
$\mathrm{S}^{+3}$ & 10.5 & 90 & $2.40(-6)$ & 1.0 & $6.0(-6)$ \\
$\mathrm{Ar}^{++}$ & 8.99 & 15.6 & $1.48(-6)$ & & \\
$\mathrm{Ar}^{++}$ & 7135 & 16.6 & $1.25(-6)$ & & \\
$\mathrm{Ar}^{+3}$ & 4740 & 1.5 & $3.22(-7)$ & 1.0 & $1.6(-6)$ \\
$\mathrm{Cl}^{+}$ & 14.3 & 0.060 & $1.1(-8)$ & & \\
$\mathrm{Cl}^{++}$ & 5538 & 0.95 & $1.01(-7)$ & & \\
$\mathrm{Cl}^{+3}$ & 11.8 & 0.308 & $1.77(-8)$ & 1.0 & $1.2(-7)$ \\
$\mathrm{P}^{++}$ & 17.9 & 0.814 & $3.42(-8):$ & $2.0:$ & $7.0(-8):$ \\
\hline
\end{tabular}

$\dagger$ Intensities given with respect to $\mathrm{H} \beta=100$.

: Indicates uncertain value.

Table 13. Comparison of abundances in NGC 3242.

\begin{tabular}{lrrrrrr}
\hline \hline Elem. & Present & $\mathrm{T}^{\dagger}$ & $\mathrm{H}^{\dagger}$ & $\mathrm{HK}^{\dagger}$ & $\mathrm{B}^{\dagger}$ & Solar $^{\dagger}$ \\
\hline $\mathrm{He}$ & 0.092 & 0.10 & 0.081 & 0.11 & 0.091 & 0.098 \\
$\mathrm{C}(-4)$ & 1.95 & & 4.12 & & 2.6 & 2.5 \\
$\mathrm{~N}(-4)$ & 1.35 & 0.34 & 0.61 & 0.49 & 0.89 & 0.84 \\
$\mathrm{O}(-4)$ & 3.8 & 3.31 & 3.38 & 4.1 & 4.35 & 4.6 \\
$\mathrm{Ne}(-5)$ & 9.0 & 7.8 & 5.1 & 8.6 & 11.0 & 12 \\
$\mathrm{~S}(-6)$ & 2.8 & 2.38 & & 2.1 & 3.2 & 14 \\
$\mathrm{Ar}(-6)$ & 1.7 & 0.98 & & 1.5 & 1.4 & 4.2 \\
$\mathrm{Cl}(-7)$ & 0.70 & 0.88 & & 1.1 & & 3.5 \\
$\mathrm{P}(-8)$ & 4.5 & & & & & 23 \\
\hline
\end{tabular}

$\dagger$ References: T: Tsamis et al. (2003), H: Henry et al. (2000), HK: Henry et al. (2004), B: Barker (1985), Solar: Asplund et al. (2005), except Ne and $\operatorname{Ar}$ (see Pottasch \& Bernard-Salas 2006).

intensities. The advantage in using the infrared measurements is quite clear.

\section{Discussion of stellar evolution}

For both nebulae there is a general agreement with the (more uncertain) earlier values. Helium, oxygen and nitrogen abundances are essentially solar. This is probably true of neon as well. On the other hand, sulfur, argon, chlorine and phosphorus are all significantly lower than solar by a factor of 3 to 4, both in NGC 6369 and NGC 3242. This is also remarkably similar to most elliptical PNe which have been studied in the infrared and which are at approximately the same galactocentric distance. The abundances of these nebulae are given in Table 15. These nebulae not only have similar morphology, but are also all at rather high
Table 14. Comparison of abundances in NGC 6369.

\begin{tabular}{lrrrrr}
\hline \hline Elem. & Present & $\mathrm{M}^{\dagger}$ & $\mathrm{P}^{\dagger}$ & $\mathrm{AK}^{\dagger}$ & Solar $^{\dagger}$ \\
\hline $\mathrm{He}(-1)$ & 1.02 & 1.14 & 1.14 & 1.3 & 0.98 \\
$\mathrm{~N}(-5)$ & 7.9 & 10 & 2.2 & 12 & 8.4 \\
$\mathrm{O}(-4)$ & 4.0 & 6.1 & 4.7 & 2.9 & 4.6 \\
$\mathrm{Ne}(-5)$ & 9.7 & 6.5 & 9.4 & 4.0 & 12 \\
$\mathrm{~S}(-6)$ & 6.0 & 7.5 & & 6.0 & 14 \\
$\mathrm{Ar}(-6)$ & 1.6 & & & 4.2 & 4.2 \\
$\mathrm{Cl}(-7)$ & 1.2 & & & 2.6 & 3.5 \\
$\mathrm{P}(-8)$ & 7.0 & & & & 23 \\
\hline
\end{tabular}

$\dagger$ References: M: Monteiro et al. (2004), P: Pena et al. (2001), AK: Aller \& Keyes (1987), Solar: Asplund et al. (2005), except Ne and Ar (see Pottasch \& Bernard-Salas 2006).

Table 15. Comparison of abundances in several elliptical PNe.

\begin{tabular}{lrrrrrrr}
\hline \hline Elem. & $3242^{\dagger}$ & $6369^{\dagger}$ & $2392^{\dagger}$ & $6826^{\dagger}$ & $7662^{\dagger}$ & $418^{\dagger}$ & $2022^{\dagger}$ \\
\hline $\mathrm{He}(-1)$ & 0.92 & 1.02 & 0.80 & 0.95 & 0.88 & & 1.06 \\
$\mathrm{C}(-4)$ & 1.95 & & 3.3 & 5.3 & 3.6 & 6.2 & 3.7 \\
$\mathrm{~N}(-4)$ & 1.35 & 0.79 & 1.85 & 0.50 & 0.67 & 0.95 & 0.99 \\
$\mathrm{O}(-4)$ & 3.8 & 4.0 & 2.9 & 4.0 & 4.2 & 3.5 & 4.7 \\
$\mathrm{Ne}(-5)$ & 9.0 & 9.7 & 8.5 & 15 & 6.4 & 8.8 & 13.4 \\
$\mathrm{~S}(-6)$ & 2.8 & 6.0 & 5.0 & 2.8 & 6.6 & 4.4 & 6.3 \\
$\mathrm{Ar}(-6)$ & 1.7 & 1.6 & 2.2 & 1.4 & 2.1 & 1.8 & 2.7 \\
$\mathrm{Cl}(-7)$ & 0.70 & 1.2 & 1.3 & 0.9 & & 1.2 & 1.3 \\
$\mathrm{P}(-8)$ & 4.5 & 7.0 & 6.5 & 17 & & & \\
$\mathrm{Fe}(-7)$ & & & 8.0 & 6.1 & & & \\
\hline
\end{tabular}

$\dagger$ References: NGC 3242 and 6369: this paper, NGC 2392: Pottasch et al. (2008), NGC 6826: Surendiranath et al. (2008), NGC 7662: Pottasch et al. (2001), IC 418: Pottasch et al. (2004), NGC 2022: Pottasch et al. (2005).

galactic latitude. The last five PNe listed all have galactic latitudes between $10^{\circ}$ and $20^{\circ}$, which is much higher than the average value. Part of this difference occurs because these are all relatively nearby nebulae. But the main reason for the high galactic latitude is probably that these nebulae are formed from stars which initially had a relatively low mass.

In considering all the nebulae in Table 15 the following is noted. The low helium abundance indicates that no helium has been produced which implies that the second dredge-up and hotbottom burning have not taken place. For some PNe the carbon abundance is somewhat higher than oxygen suggesting that the third dredge-up has taken place in these nebulae. Following the models of Karakas (2003) for the case $Z=0.008$ (which is close to the solar abundance) the increase in the carbon abundance will occur at a stellar mass of about $1.7 M_{\odot}$. The lower mass models for which the carbon has not yet increased show an increased nitrogen abundance, sometimes by a factor of two or three. When the increased carbon is found the nitrogen is predicted to have its original (solar) value. This seems to be exactly what is observed. Of the nebulae listed in Table 15 we therefore predict that NGC 3242, NGC 7662 and NGC 2022 have descended from stars with a mass of between $0.8 M_{\odot}$ and $1.5 M_{\odot}$ and NGC 6826 , NGC 2392 and IC 418 are descendents of stars from between 1.6 $M_{\odot}$ and $1.9 M_{\odot}$. Above this mass Karakas predicts an increased neon abundance by more than a factor of two, which is not seen. NGC 6369 is harder to classify since no carbon has yet been measured. From the low nitrogen abundance we tentatively conclude that this nebula is probably in the second (high mass) group. 
Notice that for all the nebulae listed in Table 15 the elements $\mathrm{S}, \mathrm{Ar}, \mathrm{Cl}, \mathrm{P}$ and $\mathrm{Fe}$ are underabundant compared to the Sun. There are several possible reasons for this. First the solar abundances may be wrong. Secondly our determinations of the abundances may be wrong. Thirdly the elements may be tied up in the dust grains in the nebula. We do not believe that our determination of the abundances can be so badly wrong. The worst case is $\mathrm{P}$ because only a single ionization stage is observed and a reasonably large correction for the missing stages of ionization has been made. It seems unlikely that this has been so poorly done. For S, Ar and $\mathrm{Cl}$ the solar abundance is somewhat uncertain and could be wrong. This is certainly the case for Ar for which a coronal value must be used. Furthermore the difference between the nebular value and the solar value is only a factor of two to three for these 3 elements. For $\mathrm{S}$ and $\mathrm{Cl}$ it is not possible to say whether the difference is due to wrong solar values or that the elements are in taken up in the dust. Ar is a noble gas and it is unlikely that it can be present in dust. For $\mathrm{P}$ and Fe the difference with the solar value is so large that these elements must be in the form of dust. Since iron has been known to be depleted in all PNe in which it has been observed, there has been some speculation in the literature as to whether iron molecules have been observed in the infrared continuum. Hony et al. (2002) claim to have detected FeS in two PNe. These authors also note that iron sulfides are abundantly found in meteoritic material. Iron could also be in the form of $\mathrm{FeO}$. But iron molecules are easily destroyed. Iron is highly refractory and should easily stick to carbonaceous dust grains which are present in PNe and are responsible for their IR continuum. Measuring the iron content of the dust grains is very difficult and has not yet been done. But it is significant that about $1 \%$ of the nebular mass must be contained in dust containing this iron molecule assuming that the original composition was solar. Notice that the spectrum of dust does not show any hydrocarbon (PAH) emission bands in the oxygen-rich nebulae listed in Table 15. Very weak PAH features are seen in the carbon-rich nebula IC 418 and in NGC 6369.

\section{The central star}

\subsection{Stellar temperature}

As discussed in the introduction, the spectrum of the central star of NGC 3242 has been studied by several authors. Pauldrach et al. (2004) have fitted model atmospheres to the measured ultraviolet stellar spectrum and Kudritzki et al. (1997) have studied the optical spectrum. Pauldrach et al. (2004) determined the effective stellar temperature from the FeIV/FeV ionization balance to be $75000 \mathrm{~K}$ while Kudritzki et al. (1997) using the ionization equilibrium of HeI and HeII also obtained a value of $75000 \mathrm{~K}$. Neither of these authors has studied the spectrum of the central star of NGC 6369. This star is of the Wolf-Rayet type and was originally classified as WC4 (see Acker et al. 1992). More recently it is classified as WO3 (Acker \& Neiner 2003). The stellar temperature associated with this spectral type is usually about $85000 \mathrm{~K}$ to $90000 \mathrm{~K}$, which is often determined by the Zanstra temperature (Acker \& Neiner 2003). This at first sight it would appear that the central star of NGC 6369 has the higher temperature of the two. The Zanstra temperatures give a slightly different picture. The hydrogen Zanstra temperature $T_{\mathrm{z}}(\mathrm{H})=69000 \mathrm{~K}$ for NGC 6369 and $57000 \mathrm{~K}$ for NGC 3242 while the ionized helium Zanstra temperature is $71000 \mathrm{~K}$ for NGC 6369 and $90000 \mathrm{~K}$ for NGC 3242. Since the ionized helium Zanstra temperature is often taken as representative of the actual temperature this would imply that NGC 3242 is the hotter star. This is a direct result of the much stronger He II line in the latter nebula. This is also reflected in the ionic distribution of the other elements. For example, $10 \%$ of the oxygen is in the form of $\mathrm{O}^{+3}$ in NGC 3242 while only $1 \%$ of the oxygen is in this ionization stage in NGC 6369.

The stellar temperature can also be estimated from the energy balance temperature of the central star. This method makes use of the fact that the average excess energy per ionizing photon (hence the temperature) can be found from the ratio of collisionally excited lines to $\mathrm{H} \beta$. For NGC 3242 this can be found from the sum of the values given in Tables 1, 4, and 6. Unobserved lines must be estimated usually from predicted ratios of observed lines. For NGC 6369 it is the sum of values given in Tables 3 and 5 again corrected for unobserved lines. For this latter nebula no ultraviolet measurements are available. For NGC 3242 the ultraviolet measurements are about $20 \%$ of the total and because the spectra of the two nebulae are so similar we will assume this to be the same in NGC 6369. This value of the ratio of the summation $I / \mathrm{H} \beta$ is about 25 or 26 in both cases. This could be slightly higher if there are important unmeasured lines. To convert this value to a stellar temperature it is not possible to use a simple equation because the result, at least for higher temperatures, depends on the amount of helium present in the nebula, its ionization state, the opacity in the nebula and ultraviolet spectral distribution of the exciting star. To obtain a temperature the formulation of Preite-Martinez \& Pottasch (1983) is used, assuming blackbody radiation from the central star. This leads to a value for the energy balance temperature $\left(T_{\mathrm{z}}(\mathrm{EB})=70000 \mathrm{~K}\right.$ in both cases. If a model atmosphere had been used instead of a blackbody, the energy balance temperature could be lower. When the three values of temperature are compared for NGC 6369 they are consistent and we adopt a temperature $T=70000 \mathrm{~K}$ for the central star of NGC 6369. Because of the higher ionization state in NGC 3242, the temperature of the central star of this nebula must be somewhat higher since it cannot be explained by a lower density. A temperature $T=80000 \mathrm{~K}$ is used because of the higher ionized helium Zanstra temperature.

If the distance to these nebulae is known the radius and luminosity of the central star may be found. For NGC 3242 an expansion distance has been measured by Hajian et al. (1995) and corrected by Mellema (2004) to a somewhat uncertain value $d=550$ pc. The distance to NGC 6369 has only been determined stastisically and thus is quite uncertain; we shall use a value of $d=1 \mathrm{kpc}$ which is an average value of the statistical distances listed in Acker et al. (1992). The nebula could be somewhat closer because it is the third brightest PNe after correction for extinction. The visual magnitudes for the central stars are $m_{\mathrm{v}}=12.43$ and 15.91 for NGC 3242 and NGC 6369 respectively. When corrected for the extinction found in Sec. 3 these values are $m_{\mathrm{v}}=11.86$ and 11.42 , which leads to radii of $0.141 R_{\odot}$ and $0.336 R_{\odot}$ respectively. Combined with the above values of central star temperature, the luminosities of these stars are $730 L_{\odot}$ and $2430 L_{\odot}$ for NGC 3242 and NGC 6369. These values of luminosity are considerably lower than found in the recent literature. For NGC 3242 Pauldrach et al. (2004) find a value of $3200 L_{\odot}$ while Kudritzki et al. (1997) find $10000 L_{\odot}$. For NGC 6369 Monteiro et al. (2004) find $8100 L_{\odot}$, but in this case much of the difference is due to the larger distance used by these authors.

\section{Discussion and conclusions}

The nebular abundances of nine elements have been determined for the PN NGC 3242; abundances of the same elements, except for carbon, have been found for NGC 6369. Both nebulae are classified as having an elliptical shape. The abundances found 
for both nebulae are very similar. For helium, oxygen and neon they are essentially the same as in the Sun. This is consistent with the expectation that these PNe are rather local. This is because the gradient in the stellar abundances as a function of distance from the galactic center (e.g. see Pottasch \& Bernard-Salas 2006) would lead to the expectation that a different abundance prevails when the position of these PNe is non-local.

The state of evolution of these nebulae is considered in conjunction with five other local PNe with elliptical shape. A comparison is made between the observed abundances and those expected using the stellar evolution models calculated by Karakas (2003). This comparison leads to the conclusion that NGC 3242 must be a descendent of a low mass star, probably between $1 M_{\odot}$ and $1.5 M_{\odot}$. NGC 6369 probably has a slightly higher initial mass, but this conclusion is less certain because the abundance of carbon is unknown.

The stellar temperature and luminosity is considered with the help of the Zanstra method, the Energy Balance method and the observed nebular excitation. For NGC 3242 we find $T_{\mathrm{s}}=80000 \mathrm{~K}$ and $L=730 L_{\odot}$ and for NGC $6369 T_{\mathrm{s}}=70000 \mathrm{~K}$ and $L=2400 L_{\odot}$. The temperatures are in quite good agreement with what is given in the literature. The luminosities, which are distance dependent, are considerably lower than given by the theories of Kudritzki et al. (1997) or Pauldrach et al. (2004). This has already been discussed by Napiwotzki (2006) in a general way and by Surendiranath \& Pottasch (2008) for the case of NGC 6826. This is evidence that all of the nebulae listed in Table 15 have central stars of quite low luminosity, probably between $500 L_{\odot}$ and $2000 L_{\odot}$.

The abundances of the other elements, $\mathrm{S}, \mathrm{Cl}, \mathrm{P}$ and $\mathrm{Fe}$ are all lower than solar. For the first two of these elements the difference with respect to the sun is a factor of two to three. In this case we cannot distinguish between the possibility that this is caused by a poor determination of the solar abundance or by the possibility that important amounts of these elements have condensed in the form of dust. For the remaining elements, $\mathrm{P}$ and Fe, the underabundance with respect to the Sun is larger; it is likely that both of these elements have formed molecules which have condensed in the form of dust. This is probably true for all the PNe listed in Table 15.

Acknowledgements. This work is based on observations made with the Spitzer Space Telescope, which is operated by the Jet Propulsion Laboratory, California Institute of Technology under NASA contract 1407. Support for this work was provided by NASA through Contract Number 1257184 issued by JPL/Caltech. We acknowledge that the IRS data on NGC 6369 has been taken from one of the GTO programs of Dale Cruikshank.

\section{References}

Acker, A., \& Neiner, C. 2003, A\&A, 403, 659

Acker, A., Marcout, J., Ochsenbein, F., et al. 1992, Strasbourg-ESO catalogue Aller, L. H., \& Czyzak, S. J. 1979, Ap\&SS, 62, 397

Aller, L. H., \& Keyes, C. D. 1987, ApJS, 65, 405

Asplund, M., Grevesse, N., \& Sauval, A. J. 2005, ASP Conf. Ser. 336, ed. T. G. Barnes III \& F. N. Bash, 25

Barker, T. 1985, ApJ, 294, 193

Becker, R. H., White, R. L., \& Edwards, A. L. 1991, ApJS, 75, 1

Benjamin, R. A., Skillman, E. D., \& Smits, D. P. 1999, ApJ, 514, 307

Bernard, S. J., Pottasch, S. R., Beintema, D. A., \& Wesselius, P. R. 2001, A\&A, 367,949

Bernard-Salas, J., Pottasch, S. R., Gutenkunst, S., Morris, P. W., \& Houck, J. R. 2008, ApJ, 672, 274

Cahn, J. H., Kaler, J. B., \& Stanghellini, L. 1992, A\&AS, 94, 399

Fluks, M. A., Plez, B., de Winter, D., et al. 1994, A\&AS, 105, 311

Gathier, R., \& Pottasch, S. R. 1988, A\&A, 197, 266

Griffith, M. R., Wright, A. E., Burke, B. F., et al. 1994, ApJS, 90, 179

Hajian, A. R., Terzian, Y., \& Bignell, C. 1995, AJ, 85, 2600

Henry, R. B. C., Kwitter, K. B., \& Bates, J. A. 2000, ApJ, 531, 928

Henry, R. B. C., Kwitter, K. B., \& Balick, B. 2004, AJ, 127, 2284

Higdon, S. J. U., Devost, D., Higdon, J. L., et al. 2004, PASP, 116, 975

Hony, S., Bouwman, J., Keller, L. P., et al. 2002, A\&A, 393, L103

Houck, J. R., Appelton, P. N., Armus, L., et al. 2004, ApJS, 154, 18

Hummer, D. G., \& Storey, P. J. 1987, MNRAS, 224, 801

Karakas, A. I. 2003, Thesis, Monash Univ. Melbourne

Kerber, F., Mignani, R. P., Guglielmetti, F., et al. 2003, A\&A, 408, 1029

Kingsburgh, R. L., \& Barlow, M. J. 1994, MNRAS, 271, 257

Krabbe, A. C., \& Copetti, M. V. F. 2006, A\&A, 450, 159

Kudritzki, R.-P., Mendez, R. H., Puls, J., \& McCarthy, J. K. 1997, IAU Symp., 180,64

Liu, X.-W., Barlow, M. J., Cohen, M., et al. 2001, MNRAS, 323, 343

Mellema, G. 2004, A\&A, 416, 623

Milne, D. K., \& Aller, L. H. 1975, A\&A, 38, 183

Monteiro, H., Schwarz, H. E., Gruenwald, R., et al. 2004, ApJ, 609, 194

Napiwotzki, R. 2006, A\&A, 451, L27

Pauldrach, A. W. A., Hoffmann, T. L., \& Mendez, R. H. 2004, A\&A, 419, 1111

Pena, M., Stasinska, G., \& Medina, S. 2001, A\&A, 367, 983

Phillips, J. P. 2003, MNRAS, 344, 501

Porter, R. L., Bauman, R. P., Ferland, G. J., et al. 2005, ApJ, 622, L73

Pottasch, S. R. 1984, Planetary Nebulae (Dordrecht: Reidel Publ. Co.)

Pottasch, S. R., \& Beintema, D. A. 1999, A\&A, 347, 974

Pottasch, S. R., \& Bernard-Salas, J. 2006, A\&A, 457, 189

Pottasch, S. R., Beintema, D. A., \& Feibelman, W. A. 2000, A\&A, 363, 767

Pottasch, S. R., Beintema, D. A., Bernard S. J., \& Feibelman, W. A. 2001, A\&A, 380,684

Pottasch, S. R., Bernard-Salas, J., Beintema, D. A., \& Feibelman, W. A. 2004, A\&A, 423, 593

Pottasch, S. R., Beintema, D. A., \& Feibelman, W. A. 2005, A\&A, 436, 965

Pottasch, S. R., Bernard-Salas, J., \& Roellig, T. L. 2008, A\&A, 481, 393

Preite-Martinez, A., \& Pottasch, S. R. 1983, A\&A, 126, 31

Seaton, M. J. 1979, MNRAS, 187, 73

Surendiranath, R., \& Pottasch, S. R. 2008, A\&A, 483, 519

Terzian, Y. 1997, IAU Symp., 180, ed. H. J. Habing, \& H. Lamers, 29,

Tinkler, C. M., \& Lamers, H. J. G. L. M. 2002, A\&A, 384, 987

Tsamis, Y. G., Barlow, M. J., Liu, X.-W., et al. 2003, MNRAS, 345, 181

Werner, M., Roellig, T. L., Low, F. J., et al. 2004, ApJS, 154, 1 